\title{
Small-Signal Modeling of PWM Dual-SEPIC DC-DC Converter by Circuit Averaging Technique
}

\author{
Agasthya Ayachit*, Alberto Reatti ${ }^{\dagger}$, and Marian K. Kazimierczuk* \\ * Department of Electrical Engineering, Wright State University, Dayton, Ohio, USA 45435 \\ \{ayachit.2, marian.kazimierczuk\}@wright.edu \\ ${ }^{\dagger}$ Department of Information Engineering, University of Florence, Florence, Italy 50139 \\ alberto.reatti@unifi.it
}

\begin{abstract}
This paper presents the small-signal modeling of a dual-SEPIC dc-dc converter power stage operating in continuousconduction mode using the circuit averaging technique. The converter component parasitic resistances are taken into account. From the small-signal model, the following expressions of the converter required to design the outer voltage loop are derived: duty cycle-to-output voltage and input-to-output voltage transfer functions, input impedance, and output impedance. A design example is given and the characteristics of these transfer functions are analyzed. Simulations are performed to verify the correctness of the theoretically obtained transfer functions. The dual-SEPIC converter has four reactive components and yields transfer functions of order two. A brief discussion on this aspect is also provided.
\end{abstract}

\section{INTRODUCTION}

A dual-SEPIC (zeta) converter is the dual to the SEPIC (single ended primary inductance converter) converter [1]. These topologies have found its application in various areas such as electronic ballasts, power factor correctors, battery chargers, etc. [1] - [5]. Designing control circuits for SEPIC converters is a challenge since their transfer functions are of fourth order [3] - [5]. However, by inspecting the operation of the dual-SEPIC converter, state equations of order two were obtained, forming a motive to develop its small-signal model and hence derive the network functions.

The dual-SEPIC dc-dc converter shown in Fig. 1 is considered in this paper. The main objectives of this paper are: (a) to develop the dc model using circuit averaging technique by averaging the steady-state switch current and diode voltage waveforms [6] - [8], (b) to develop a small-signal linear model, (c) to derive the expressions for the open-loop small-signal power stage transfer functions, input and output impedances, (d) to validate the theoretical predictions through simulations, and (e) to justify how a circuit with four reactive components is described by second-order transfer functions.

This paper is organized as follows. In Section II, the fundamental steady-state equations required for circuit averaging is presented. The dc, averaged model, the large-signal model, and the low-frequency small-signal linear equivalent model are developed in this Section. Using the small-signal model, the relevant transfer functions are derived in Section III. Section IV presents a design example of the dual-SEPIC dcdc converter and the theoretical results are validated using

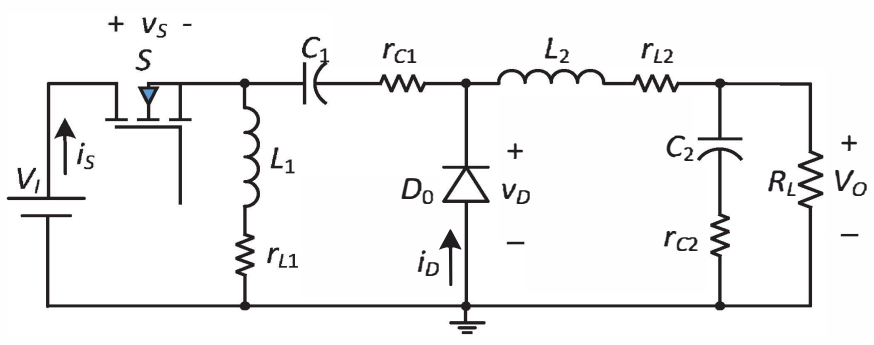

Fig. 1. Circuit of the dual-SEPIC dc-dc converter including inductor and capacitor parasitic resistances.
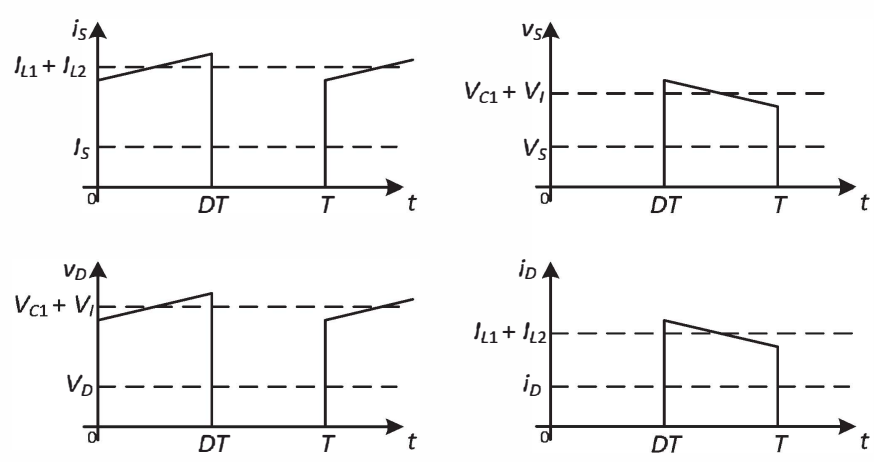

(a)

(b)

Fig. 2. Key waveforms of the switching network. (a) Switch current and diode voltage during $0<t \leq D T$. (b) Switch voltage and diode current waveforms during $D T<t \leq \bar{T}$.

simulations. Finally, a few concluding remarks are presented in Section V.

\section{MODEl DeVElopMent}

\section{A. Averaged Circuit Model}

Fig. 2 shows the waveforms of the MOSFET current $i_{S}$, diode current $i_{D}$, MOSFET voltage $v_{S}$, and diode voltage $v_{D}$. The waveforms comprise of both dc or averaged and a switching frequency components. Using the circuit averaging technique, the dc or low-frequency equivalent of the dualSEPIC converter shown in Fig. 1 is obtained. The circuit averaging technique involves averaging the switch currents and 


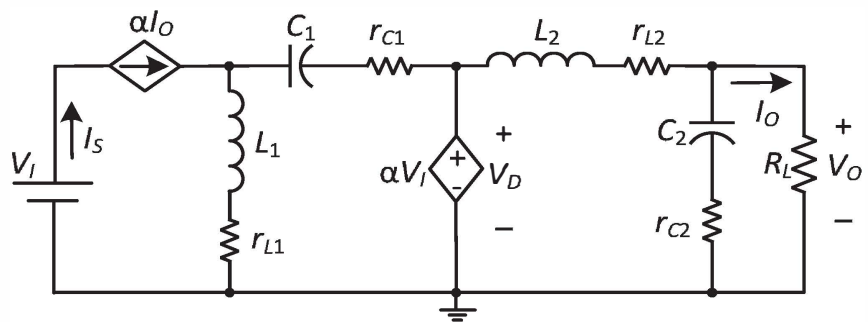

Fig. 3. Averaged, dc equivalent model of the dual-SEPIC converter in CCM, where $\alpha=\frac{D}{D^{\prime}}$.

voltages over a single switching time period. The expression for average current through the MOSFET $S$ is

$I_{S}=\frac{1}{T} \int_{0}^{T} i_{S} d t=\frac{1}{T} \int_{0}^{D T}\left(I_{L 1}+I_{L 2}\right) d t=D\left(I_{L 1}+I_{L 2}\right)$,

where $D$ is the duty cycle of the MOSFET. The expression for average current through the diode $D_{0}$ is

$I_{D}=\frac{1}{T} \int_{0}^{T} i_{D} d t=\frac{1}{T} \int_{D T}^{T}\left(I_{L 1}+I_{L 2}\right) d t=D^{\prime}\left(I_{L 1}+I_{L 2}\right)$,

where $D^{\prime}=1-D$. Therefore, from (1) and (2), the average MOSFET current is

$$
I_{S}=\frac{D}{D^{\prime}} I_{D}=\frac{D}{D^{\prime}} I_{L 2}
$$

During the diode conduction interval, the average diode current $I_{D}$ is equal to the average current through the inductor $L_{2}$ is $I_{L 2}$ equal to the de load current $I_{O}[1]$, i.e.,

$$
I_{D}=I_{L 2}=I_{O}=\frac{V_{O}}{R_{L}} \text {. }
$$

Similarly, the expression for average voltage across the diode $D_{0}$ is

$$
V_{D}=\frac{1}{T} \int_{0}^{T} v_{D} d t=\frac{1}{T} \int_{D T}^{T}\left(V_{C 1}+V_{I}\right) d t=D^{\prime}\left(V_{C 1}+V_{I}\right)
$$

and the average voltage across the MOSFET is

$$
V_{S}=\frac{1}{T} \int_{0}^{T} v_{S} d t=\frac{1}{T} \int_{0}^{D T}\left(V_{C 1}+V_{I}\right) d t=D\left(V_{C 1}+V_{I}\right)
$$

Thus, from (5) and (6), we have

$$
V_{D}=\frac{D}{D^{\prime}} V_{S}=\frac{D}{D^{\prime}} V_{I}
$$

Equations (3) and (7) are the expressions for the averaged MOSFET current and diode voltage. The switching network can be replaced by controlled current and voltage sources [6], [7]. Fig. 3 shows the averaged, dc equivalent model of the dual-SEPIC converter with its MOSFET replaced by a currentcontrolled current source and the diode by a voltage-controlled current source. The load resistance can be represented as a constant current sink with an average value $I_{O}$.

\section{B. Small-Signal Equivalent Model}

The averaged current and voltage in (3) and (7) can be expressed as slowly varying and time-dependent large-signal quantities. The large-signal quantities relevant to the averaged switching network are $d_{T}, i_{D}, i_{S}, v_{D}, v_{S}$ and represented as

$$
\begin{array}{r}
d_{T}=D+d, \\
i_{L 2}=I_{L 2}+i_{l 2}, \\
i_{S}=I_{S}+i_{s}, \\
v_{D}=V_{D}+v_{d}, \\
v_{I}=V_{I}+v_{i},
\end{array}
$$

where $D, I_{D}, I_{S}, V_{D}$, and $V_{I}$ are the dc components, while $d, i_{d}, i_{s}, v_{d}$, and $v_{i}$ are the small-signal ac components. Thus, the expressions for the large-signal MOSFET current and the large-signal diode voltage are

$$
\begin{aligned}
& i_{S}=\frac{d_{T}}{d_{T}^{\prime}} i_{L 2}, \\
& v_{D}=\frac{d_{T}}{d_{T}^{\prime}} v_{I},
\end{aligned}
$$

where $d_{T}^{\prime}=1-d_{T}=D^{\prime}-d$. Substituting (8) into (9), we get

$$
D^{\prime} I_{S}+D^{\prime} i_{s}-I_{S} d-d i_{s}=D I_{L 2}+D i_{l 2}+I_{D} d+d i_{l 2}
$$

and

$$
D^{\prime} V_{D}+D^{\prime} v_{d}-V_{D} d-d v_{d}=D V_{S}+D v_{i}+V_{I} d+d v_{i} .
$$

Using (3) and (7) in (10) and (11) yields

$$
D^{\prime} i_{s}=I_{S} d+d i_{s}+D i_{l 2}+I_{D} d+d i_{l 2}
$$

and

$$
D^{\prime} v_{d}=V_{D} d+d v_{d}+D v_{i}+V_{I} d+d v_{i} .
$$

The principle of linearization can be applied to the expressions in (12) and (13), if and only if the following small-signality conditions are satisfied:

$$
\begin{gathered}
d<<D, \\
v_{s}<<V_{S}, \\
i_{s}<<I_{S}, \\
v_{d}<<V_{D}, \\
v_{i}<<V_{I} .
\end{gathered}
$$

Thus, (12) and (13) can be approximated to obtain the linear small-signal MOSFET current $i_{s}$ and small-signal diode voltage $v_{d}$ as

$$
i_{s}=\frac{I_{S}}{D^{\prime}} d+\frac{D}{D^{\prime}} i_{l 2}+\frac{I_{L 2}}{D^{\prime}} d=\frac{I_{L 2}}{D^{\prime 2}} d+\frac{D}{D^{\prime}} i_{l 2}
$$

and

$$
v_{d}=\frac{V_{D}}{D^{\prime}} d+\frac{D}{D^{\prime}} v_{i}+\frac{V_{I}}{D^{\prime}} d=\frac{V_{I}}{D^{\prime 2}} d+\frac{D}{D^{\prime}} v_{i} .
$$




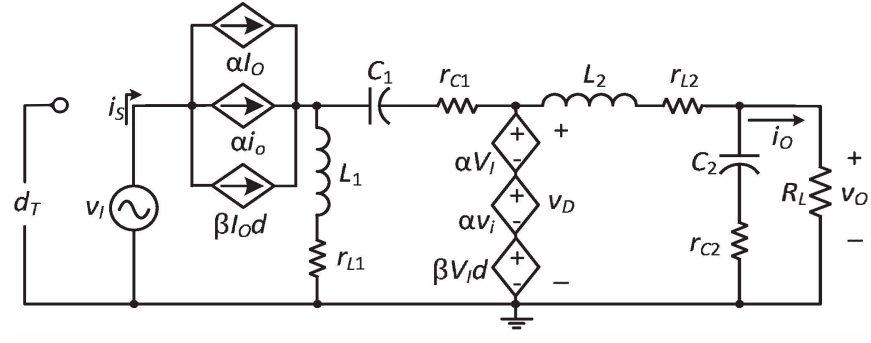

Fig. 4. Large-signal linear model of the dual-SEPIC converter in CCM, where $\alpha=\frac{D}{D^{\prime}}$ and $\beta=\frac{1}{D^{\prime 2}}$

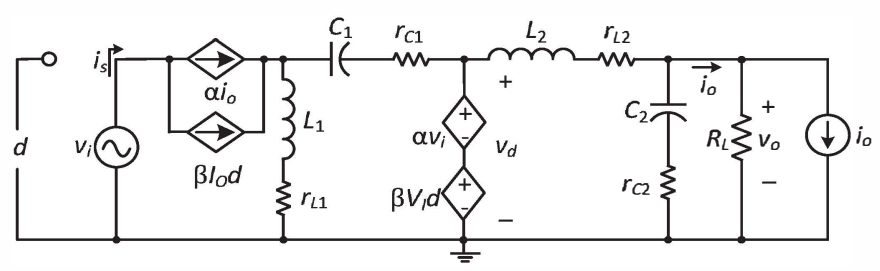

Fig. 5. Small-signal linear model of the dual-SEPIC converter in CCM, where $\alpha=\frac{D}{D^{\prime}}$ and $\beta=\frac{1}{D^{\prime 2}}$

A complete large-signal linearized model is shown in Fig. 4, where the MOSFET is replaced by both $\mathrm{dc}$ and ac current sources, while the diode is replaced by the dc and ac voltage sources. The dc model can be obtained by removing all the ac components in Fig. 4 and was shown in Fig. 3. Fig. 5 shows the small-signal linear model and is used to derive the expressions for the various power stage transfer functions of the dual-SEPIC converter in CCM.

\section{SMALL-Signal TRANSFER FunCtions}

The power stage transfer functions can be derived using the small-signal model shown in Fig. 5. The main transfer functions required for the outer loop design are: (a) duty cycleto-output $T_{p}$, (b) input-to-output $M_{v}$, (c) input impedance $Z_{i}$, and output impedance $Z_{o}$. The impedances in the model can be lumped and expressed as follows:

$$
\begin{gathered}
Z_{L 1}=r_{L 1}+s L_{1}, \\
Z_{C 1}=r_{C 1}+\frac{1}{s C_{1}}, \\
Z_{L 2}=r_{L 2}+s L_{2}, \\
Z_{L}=R_{L} \|\left(r_{C 2}+\frac{1}{s C_{2}}\right)=\frac{R_{L}\left(s r_{C 2} C_{2}+1\right)}{\left(R_{L}+r_{C 2}\right) C_{2} s+1} .
\end{gathered}
$$

\section{A. Duty Cycle-to-Output Voltage Transfer Function}

The control transfer function can be determined by letting $v_{i}$ and $i_{o}$ to zero in the small-signal model shown in Fig. 5. Using the voltage divider principle, we get

$$
v_{o}=\frac{V_{I}}{D^{\prime 2}} \frac{Z_{L}}{Z_{L}+Z_{L 2}} d
$$

to yield the duty cycle-to-output voltage transfer function as

$$
T_{p}=\frac{v_{o}}{d}=\frac{V_{I}}{D^{\prime 2}} \frac{Z_{L}}{Z_{L}+Z_{L 2}} .
$$

Substituting (17) into (19), the expression for $T_{p}$ in the standard second-order form is

$$
T_{p}=T_{p x} \frac{s+\omega_{z n}}{s^{2}+2 \xi \omega_{o} s+\omega_{o}^{2}}=T_{p 0} \frac{1+\frac{s}{\omega_{z n}}}{1+\frac{2 \xi s}{\omega_{\bullet}}+\frac{s^{2}}{\omega_{\bullet}^{2}}},
$$

where

$$
T_{p x}=\frac{V_{I} R_{L} r_{C 2}}{D^{\prime 2} L_{2}\left(R_{L}+r_{C 2}\right)},
$$

the gain at dc is

$$
T_{p 0}=\frac{V_{I}}{D^{\prime 2}} \frac{R_{L}}{r_{L 2}+R_{L}},
$$

the natural undamped corner frequency is

$$
\omega_{o}=\sqrt{\frac{r_{L 2}+R_{L}}{L_{2} C_{2}\left(r_{C 2}+R_{L}\right)}},
$$

the frequency of the zero due to the filter capacitor branch is

$$
\omega_{z}=\frac{1}{r_{C 2} C_{2}},
$$

and the damping coefficient is

$$
\xi=\frac{L_{2}+C_{2}\left[R_{L}\left(r_{C 2}+r_{L 2}\right)+r_{C 2} r_{L 2}\right]}{2 \sqrt{\left(R_{L}+r_{C 2}\right) L_{2} C_{2}}} .
$$

\section{B. Input-to-Output Voltage Transfer Function}

The equivalent model shown in Fig. 5 can be used to evaluate the input-to-output voltage transfer function of the dual-SEPIC dc-dc converter. We arrive at the required model by setting the small-signal sources $d$ and $i_{o}$ to zero. Applying the voltage divider principle at the output stage, we get

$$
v_{o}=\frac{D v_{i}}{D^{\prime}} \frac{Z_{L}}{Z_{L}+Z_{L 2}}
$$

to yield the input-to-output voltage transfer function as

$$
M_{v}=\frac{v_{o}}{v_{i}}=\frac{D}{D^{\prime}} \frac{Z_{L}}{Z_{L}+Z_{L 2}} .
$$

Substituting for the impedances given in (17) into (27) results in the audio susceptibility as

$$
M_{v}=M_{v x} \frac{s+\omega_{z}}{s^{2}+2 \xi \omega_{o} s+\omega_{o}^{2}}=M_{v 0} \frac{1+\frac{s}{\omega_{z}}}{1+\frac{2 \xi s}{\omega_{\bullet}}+\frac{s^{2}}{\omega_{\bullet}^{2}}},
$$

where

$$
M_{v x}=\frac{D R_{L} r_{C 2}}{D^{\prime} L_{2}\left(R_{L}+r_{C 2}\right)},
$$

the gain at dc is

$$
M_{v 0}=\frac{D}{D^{\prime}} \frac{R_{L}}{r_{L 2}+R_{L}} .
$$

The remainder of the parameters in (28) have been determined in (23), (24), and (25). 


\section{Input Impedance}

The equivalent model shown in Fig. 5 can be used to determine the expression for the input impedance of the dualSEPIC dc-dc converter. The small-signal perturbations $d$ and $i_{o}$ are reduced to zero. The current through the inductor $L_{2}$ is

$$
i_{l 2}=\frac{D}{D^{\prime}\left(Z_{L 2}+Z_{L}\right)} v_{i}
$$

The input current $i_{i}$ is

$$
i_{i}=\frac{D}{D^{\prime}} i_{l 2}=\left(\frac{D}{D^{\prime}}\right)^{2} \frac{v_{i}}{Z_{L}+Z_{L 2}}
$$

to yield the expression for the input impedance as

$$
\begin{array}{r}
Z_{i}=\frac{v_{i}}{i_{i}}=\left(\frac{D^{\prime}}{D}\right)^{2}\left(Z_{L}+Z_{L 2}\right) \\
=Z_{i x} \frac{s^{2}+2 \xi \omega_{o} s+\omega_{o}^{2}}{s+\omega_{p z}}=Z_{i 0} \frac{1+\frac{2 \xi s}{\omega_{o}}+\frac{s^{2}}{\omega_{o}^{2}}}{1+\frac{s}{\omega_{z z}}},
\end{array}
$$

where

$$
Z_{i x}=\left(\frac{D^{\prime}}{D}\right)^{2} L_{2} .
$$

The input resistance at $\mathrm{dc}$ is

$$
Z_{i 0}=Z_{i}(0)=R_{i 0} \approx\left(\frac{D^{\prime}}{D}\right)^{2} R_{L}
$$

and the left-half plane pole is

$$
\omega_{z z}=\frac{R_{L}}{\left(R_{L}+r_{C 2}\right) C_{2}} .
$$

\section{Output Impedance}

A test voltage signal $v_{t}$ is applied across the load resistance, which yields a small-signal current $i_{t}$. By setting the perturbations $d$ and $v_{i}$ to zero, we obtain the output impedance as

$$
Z_{o}=\frac{v_{t}}{i_{t}}=\frac{Z_{L 2} Z_{L}}{Z_{L 2}+Z_{L}} .
$$

Manipulating (38), one obtains a second-order transfer function for the output impedance, whose expression resembles that of a parallel resonant circuit. The dc gain is

$$
Z_{o}(0)=R_{o 0}=\frac{R_{L} r_{L 2}}{R_{L}+r_{L 2}} .
$$

\section{RESUlts}

\section{A. Theoretical Prediction}

A dual-SEPIC converter with the following specifications was considered [1]: $V_{I}=28 \mathrm{~V}, f_{s}=100 \mathrm{kHz}, P_{O}=60$ $\mathrm{W}$, and $V_{O}=12 \mathrm{~V}$. Using the design equations presented in [1], the values of inductors and capacitors for a nominal duty ratio of $D=0.3$ were: $L_{1}=L_{2}=120 \mu \mathrm{H}$ to ensure continuous-conduction mode, $C_{1}=16 \mu \mathrm{F}$, and $C_{2}=10 \mu \mathrm{F}$. The dc and low-frequency winding resistances of the inductors $L_{1}$ and $L_{2}$ are respectively, $r_{L 1}=r_{L 2}=10 \mathrm{~m} \Omega$. The parasitic

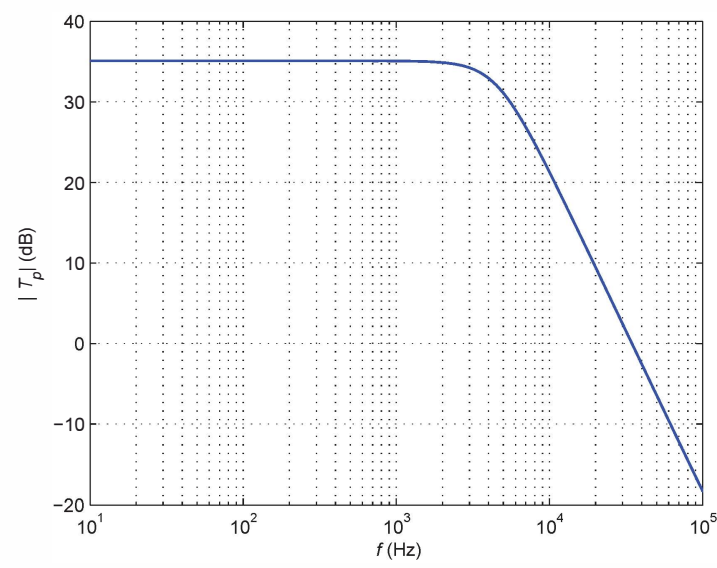

Fig. 6. Theoretically obtained magnitude plot of duty cycle-to-output voltage transfer function $T_{p}$.

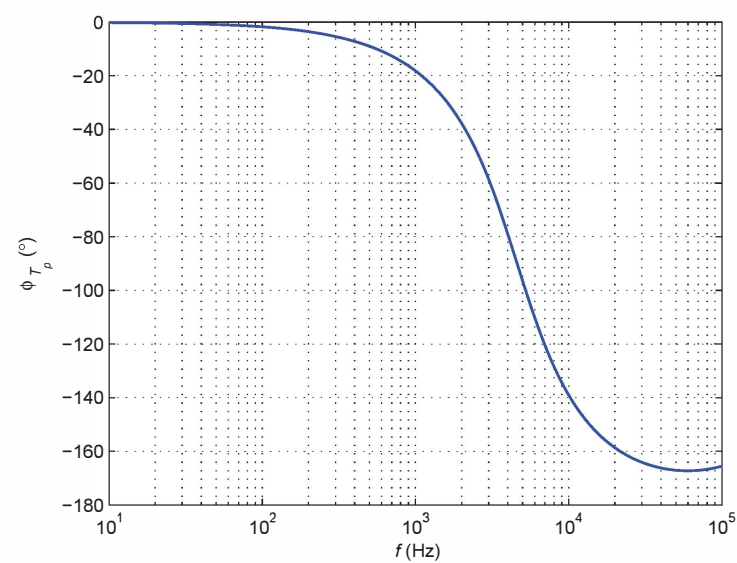

Fig. 7. Theoretically obtained phase plot of duty cycle-to-output voltage transfer function $T_{p}$.

resistances of the filter capacitors $C_{1}$ and $C_{2}$, respectively, are $r_{C 1}=r_{C 2}=30 \mathrm{~m} \Omega$.

The magnitude and phase plots of the duty cycle-to-output voltage transfer function using (20) as a function of frequency are as given in Fig. 6 and Fig. 7, respectively. A dc gain of $T_{p 0}=35.79 \mathrm{~dB}=58.14 \mathrm{~V} / \mathrm{V}$ was obtained and the undamped natural frequency was observed at $f_{o}=\omega_{o} / 2 \pi=4.5 \mathrm{kHz}$. The frequency of the left-half plane zero was $f_{z}=\omega_{z} / 2 \pi=$ $530 \mathrm{kHz}$. The assumed value of $r_{C 2}$ is very small yielding a high zero corner frequency. The damping coefficient calculated using (25) is $\xi=1.12>1$. Therefore, the magnitude plot does not exhibit an overshoot at the natural frequency. The phase plot does not cross $-180^{\circ}$ and is a characteristic of the minimum-phase systems such most of the buck-derived dcdc converters. Therefore, establishing a control technique is relatively simple. The magnitude and the phase of input-tooutput voltage transfer function $M_{v}$ with respect to frequency as given in (28) are shown in Figs. 8 and 9. The response characteristics of $M_{v}$ are similar to that of $T_{p}$ and differ only in the values of the dc gain. Fig. 10 and Fig. 11 show the magnitude and phase of the input impedance $Z_{i}$ as given in (34). At dc, the input resistance is governed by the load 


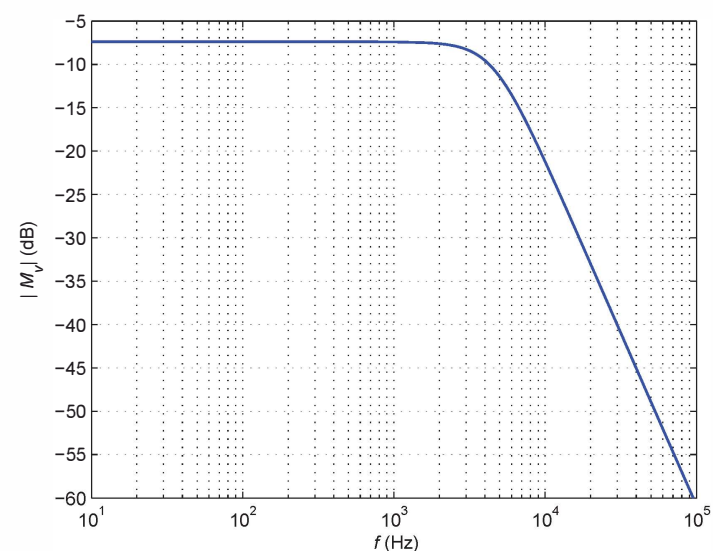

Fig. 8. Theoretically obtained magnitude plot of input-to-output voltage transfer function $M_{v}$

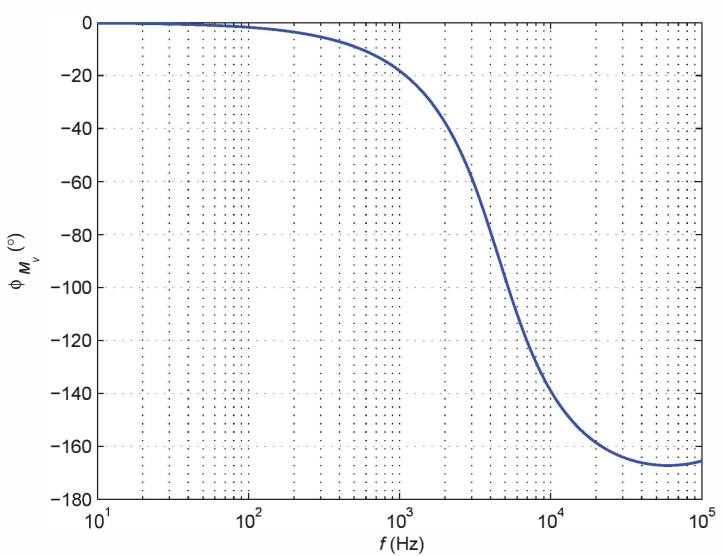

Fig. 9. Theoretically obtained phase plot of input-to-output voltage transfer function $M_{v}$.

resistance as given in (36) and is equal to $R_{i 0} \approx 13.06 \Omega$. As the frequency increases, the input impedance increases, where the reactance of $L_{2}$ is significant. From the phase plot, one may observe that the phase is zero at $\mathrm{dc}$ and reaches $90^{\circ}$ with increase in frequency showing the effect of the inductance $L_{2}$. The magnitude and phase of the small-signal output impedance of the dual-SEPIC dc-dc converter as provided in (38) are shown in Figs. 12 and 13. The value of the impedance at dc is $R_{o 0} \approx 10 \mathrm{~m} \Omega$ and agrees with (39). The output impedance increases with frequency and is inductive up to $f_{o}$. Beyond $f_{o}$, the output impedance decreases with increase in frequency and is capacitive.

\section{B. Actual Circuit Simulations}

The dual-SEPIC dc-dc converter shown in Fig. 1 was simulated on Saber circuit simulator for the values given in Section IV-A. A basic pulse-width modulator circuit was constructed. The carrier sawtooth voltage waveform with an amplitude of $V_{T m}=5 \mathrm{~V}$ and a control voltage of $V_{C}=1.5$ $\mathrm{V}$ were considered. A small-signal perturbation in the control voltage provided a small-signal variation in the duty cycle given by $d=v_{c} / V_{T m}$ [2]. Fig. 14 shows a comparison

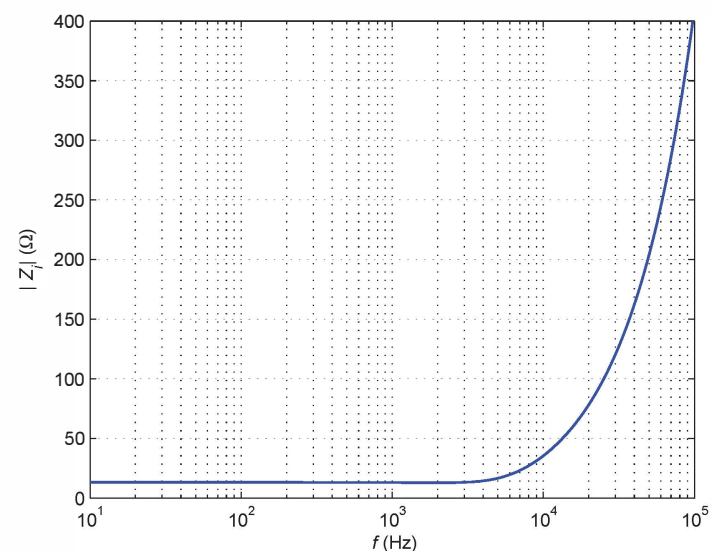

Fig. 10. Theoretically obtained magnitude plot of input impedance $Z_{i}$.

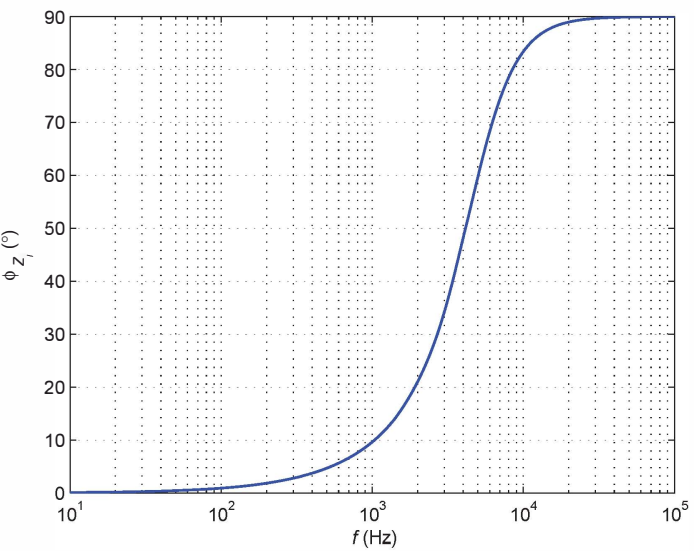

Fig. 11. Theoretically obtained phase plot of input impedance $Z_{i}$.

of theory and simulations for $T_{p}$. Subsequently, the input voltage was perturbed about the operating point $V_{I}$ by injecting a sinusoidal test voltage in series with $V_{I}$. Fig. 15 shows the comparison of theoretical and simulation results for $M_{v}$ validating the predicted analysis.

\section{Circuit with Four Reactive Components Described by Second-Order Transfer Functions}

From the circuit of dual-SEPIC dc-dc converter shown in Fig. 1, one may speculate that the circuit is of fourth order due to the presence of two pairs of inductors and capacitors. However, the transfer functions derived in Section III are of second order. Based on the circuit operation [1], during the MOSFET ON interval, only one inductor and two series capacitors are present and the overall loop is of second order. Similarly, during MOSFET OFF interval, the loop presents two inductors and two capacitors both in series yielding second order state equations. Thus, the overall transfer function is that of the second order and the dominant effect is produced by the output stage comprising of the diode $D_{0}$ and the low-pass filter $L_{2}, C_{2}, R_{L}$. It must be noted that the reduction in the order is not inherent in the SEPIC converter and the transfer functions are of fourth order [3]. 


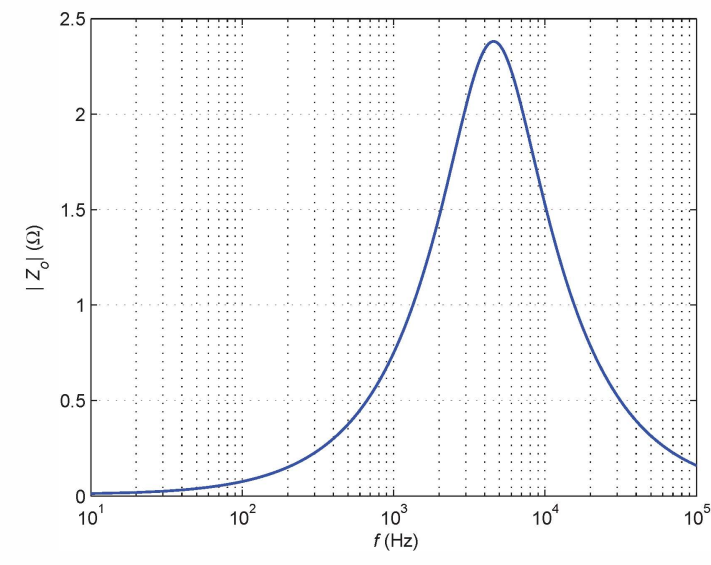

Fig. 12. Theoretically obtained magnitude plot of output impedance $Z_{O}$.

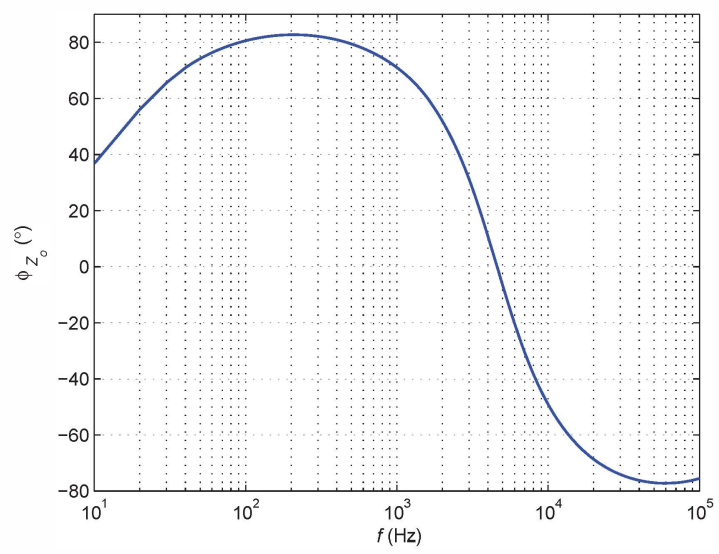

Fig. 13. Theoretically obtained phase plot of output impedance $Z_{O}$.

\section{CONCLUSiOn}

This paper has presented the derivation of a small-signal model of a dual-SEPIC dc-dc converter operating in continuous conduction mode. Using circuit averaging technique, the averaged and the large-signal models have also been developed. The following expressions have been derived: duty cycle-to-output voltage and input-to-output voltage transfer function, input impedance, and output impedance. The parasitic resistances have also been included in the model development. A design example has been considered and the properties of these transfer functions have been analyzed. Switched-circuit simulations have been performed on Saber circuit simulator to validate the correctness of the theoretically obtained transfer functions. Good agreement between the theoretical and simulation results was observed.

A practical explanation on how a dual-SEPIC dc-dc converter comprising of four reactive components yields secondorder transfer functions has been discussed. It must be noted that this phenomenon does not occur in conventional SEPIC converters or Cuk converters, where fourth-order transfer functions are obtained. Therefore, designing closed-loop circuits for the dual-SEPIC converters is comparatively easier than those for the traditional SEPIC counterparts.
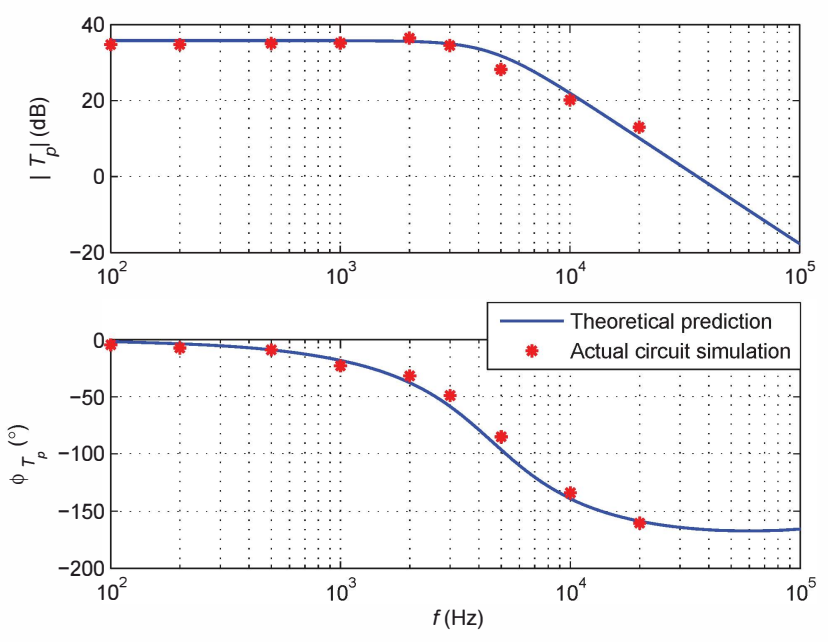

Fig. 14. Comparison of theoretical and simulation results for the duty cycleto-output voltage transfer function.
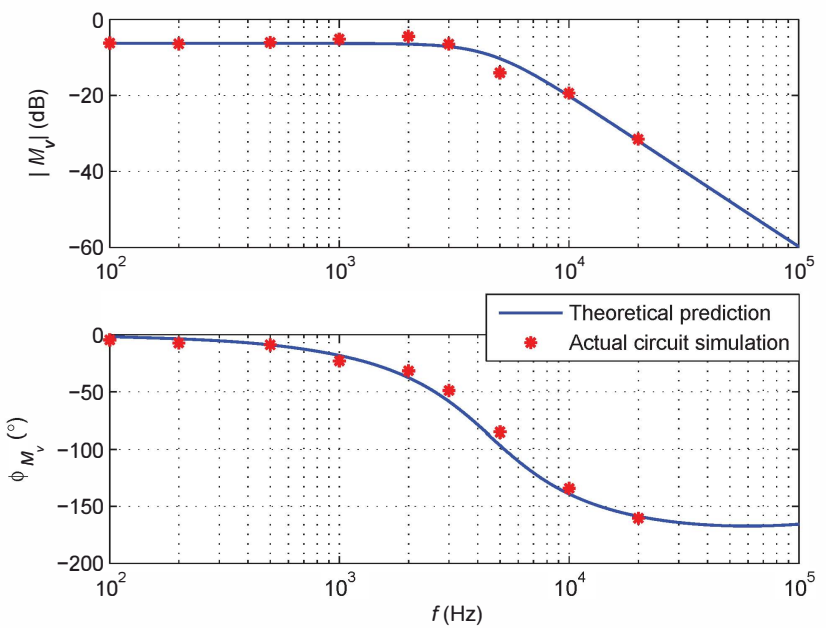

Fig. 15. Comparison of theoretical and simulation results for the input-tooutput voltage transfer function.

\section{REFERENCES}

[1] J. J. Jozwik and M. K. Kazimierczuk, "Dual Sepic PWM switchingmode DC/DC power converter," IEEE Trans. Ind. Electron., vol. 36, no. 1, Feb. 1989.

[2] M. K. Kazimierczuk, Pulse-width Modulated dc-dc Power Converters. 2nd. Ed., John Wiley \& Sons, 2015.

[3] L. K. Wong and T. K. Man, "Small signal modelling of open-loop SEPIC converters," IET Power Electron., vol. 3, no. 6, pp. 858-868, Nov. 2010.

[4] S. Hegde and A. Izadian, "A new SEPIC inverter: Small signal modeling," in Proc. IEEE Conf. Industrial Electronics Society, IECON, Vienna, Austria, 2013, pp. 240-245.

[5] J. Yao, J. Zhao and A. Abramovitz, "Modeling of the tapped inductor SEPIC converter by the TIS-SFG approach," in Proc. IEEE Conf. Industrial Electronics Society, IECON, Yokohama, Japan, 2015, pp. 1674-1679.

[6] D. Czarkowski and M. K. Kazimierczuk, "Energy-conservation approach to modeling PWM DC-DC converters," IEEE Trans. Aerosp and Electron. Syst., vol. 29, no. 3, pp. 1059-1063, Jul. 1993.

[7] M. K. Kazimierczuk and D. Czarkowski, "Application of the principle of energy conservation to modeling of PWM converters," Proc. IEEE Conf. Control Appl.,, Vancouver, Canada, Sept. 13-16, 1993, pp. 291-296.

[8] A. Ayachit and M. K. Kazimierczuk, "Analysis of switching network in PWM DC-DC converters in terms of averaged resistance and conductance," IET Letters, vol. 49, no. 11, May 2013. 